\title{
Prehospital intubation for patients with severe head injury: More is not necessarily better
}

\section{Clinical question}

Does prehospital intubation improve outcome in patients with severe head injuries?

\section{Article chosen}

Wang HE, Peitzman AB, Cassidy LD, et al. Out-of hospital endotracheal intubation and outcome after traumatic brain injury. Ann Emerg Med 2004;44:439-50.

\section{Objective}

To compare the impact of prehospital versus emergency department (ED) intubation on survival and functional status in severely head injured patients.

\section{Background}

Hypoxia is a harmful insult in the setting of traumatic brain injury. Current guidelines for acute traumatic brain injury therapy call for aggressive measures to prevent hypoxia, including the use of endotracheal intubation. ${ }^{1} \mathrm{Al}-$ though intubation is readily accomplished in the ED and hospital settings, multiple factors may complicate its performance in the out-of-hospital setting, especially in traumatic brain injury patients. Some authors have advocated advanced airway management techniques, including the use of neuromuscular blockade-assisted endotracheal intubation, for these patients. ${ }^{2}$ Previous studies have generated conflicting data about the impact of out-of-hospital endotracheal intubation in the management of traumatic brain injury, with some studies pointing to benefit ${ }^{3}$ and others suggesting harm. ${ }^{4-6}$

\section{Population studied}

In this study, all adults treated between Jan. 1, 2000, and Dec. 31, 2002, with severe traumatic brain injury — as defined by a score of $\geq 3$ using the head/neck portion of the Abbreviated Injury Scale - were considered for inclusion. This score denotes patients with head injuries considered serious, severe, critical or unsurvivable. Patients intubated out-of-hospital or in the ED were included. Patients not intubated in the prehospital setting or ED, not transported by paramedics with advanced airway skills, and those transferred from a hospital to a trauma centre were excluded.

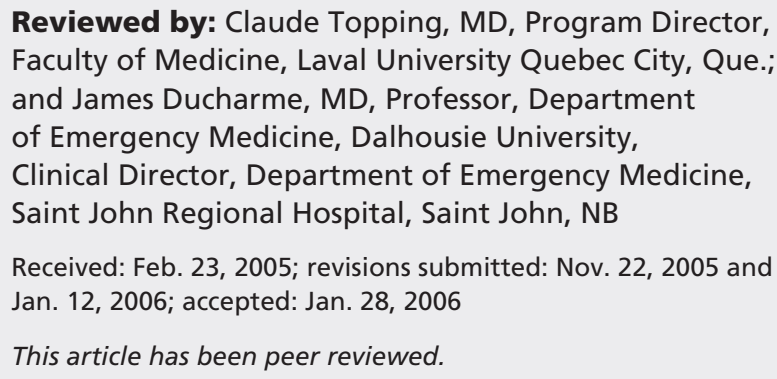

Reviewed by: Claude Topping, MD, Program Director, Faculty of Medicine, Laval University Quebec City, Que.; and James Ducharme, MD, Professor, Department of Emergency Medicine, Dalhousie University, Clinical Director, Department of Emergency Medicine, Saint John Regional Hospital, Saint John, NB

Received: Feb. 23, 2005; revisions submitted: Nov. 22, 2005 and Jan. 12, 2006; accepted: Jan. 28, 2006

This article has been peer reviewed.

Can J Emerg Med 2006;8(2):116-8

\section{Study design}

This was a retrospective review of a trauma registry database, specifically "The Pennsylvania Trauma Outcome Study" including all trauma centres and emergency medical services (EMS) systems accredited by the state of Pennsylvania.

\section{Variable studied}

Endotracheal intubation in the prehospital setting was compared with intubation performed in the ED. Airway control using the Combitube ${ }^{\mathrm{TM}}$ (Tyco-Kendall, Mansfield, Mass.) or cricothyrodotomy was considered equivalent to intubation.

\section{Primary outcome measure}

Death before hospital discharge. Secondary outcomes were neurologic and functional status at the time of discharge.

\section{Results}

Identified were 4098 patients, of whom 1797 (43.9\%) were intubated in the prehospital setting, with the remaining patients intubated in the ED. There was an absolute $20.3 \%$ mortality difference favouring ED intubation. Adjusted odds of death were higher for out-of-hospital endotracheal intubation than ED endotracheal intubation (odds ratio [OR] 3.99; 95\% confidence interval [CI] 3.21-4.93). Prehospital intubation was also associated with worse neurologic and functional outcomes: $18.2 \% \mathrm{v}$. $15.5 \%$ (relative risk [RR] 1.17; adjusted OR, 1.61; $95 \%$ CI 1.15-2.26). 


\section{Conclusions}

Patients suffering severe traumatic brain injuries who receive an out-of-hospital endotracheal intubation have higher mortality rates and worse neurologic and functional outcomes than do those intubated in the ED. Further study is required to distinguish whether it is the procedure (outof-hospital endotracheal intubation) or other associated factors that are causative.

\section{Commentary}

With the emergence of paramedics in the 1970s in the US, increasingly complex care has been offered to patients before hospital arrival. Canada has followed a similar course despite a lack of good supportive research. Two other recent studies looking at prehospital Advanced Life Support (ALS) have questioned its value. Stiell and colleagues demonstrated that Advanced Cardiac Life Support (ACLS) offered no increased value over rapid defibrillation alone in prehospital cardiac arrest. ${ }^{7}$ Gausche and coworkers showed that prehospital pediatric intubation in Los Angeles County provided no significant improvement in survival or neurologic outcome when compared with bag-valve-mask airway management. ${ }^{8}$ The decades-long debate of "scoop and run" versus "stay and play" for trauma patients remains largely unresolved.

This study suggests that, if you suffer a serious head injury in the state of Pennsylvania, you might prefer to avoid intubation until your arrival at the ED. For supporters of prehospital ALS, these results seem counter-intuitive. In theory, intubation secures the airway and protects against aspiration, allowing for ventilatory support and optimal oxygen delivery, all of which should decrease the risk of secondary insult following severe brain injury. However, this reasoning is not supported by the data at hand. The authors hypothesize that complications of out-of-hospital endotracheal intubation, including desaturation, procedurerelated bradycardia, unrecognized endotracheal tube misplacement, and non-use of neuroprotective drugs could explain the worse outcomes.

This study attempts to answer the question: "Should patients with severe traumatic brain injury be intubated in the prehospital setting?" Although a randomized controlled study would be the ideal design to obtain a definitive answer, getting ethical approval for such a trial has been cited as a barrier given that prehospital intubation is considered the standard of care by many. These authors chose what they felt was the best available methodology: analysis of a high-quality trauma registry populated by trained abstracters and maintained using quality assurance procedures. This database collected information on more than
10000 trauma victims annually. Recognizing that the 2 groups (out-of-hospital v. ED intubation) were likely different in regards to severity, several measurable confounders (severity: Abbreviated Injury Score [AIS] and systolic blood pressure; mode of transport: helicopter v. ground) were included in their multivariate analysis with odds estimates adjusted for severity. They also incorporated a propensity score as a covariate in each regression model. A propensity score is a scalar measure that was used here to summarize the effects of selected preexisting medical conditions, in-hospital occurrences and social variables (e.g., drug and alcohol use, ethnicity and insurance coverage). Propensity scores have been widely applied in the medical literature for a variety of purposes, including bias reduction, multivariable matching and covariate adjustment. ${ }^{9}$

Despite all of the above, this study has a number of key limitations. Although the authors assure the reader that they accounted for differences in severity between the prehospital- and ED-intubated patients, they did not publish unadjusted data that would allow readers to determine why the mortality risk increases from 2.39 (univariate) to 3.99 in the adjusted analysis. This is an unexpected finding because, if the prehospital group was in fact more severe we would expect the opposite. We are told that, of 9720 patients with severe head injuries, 5132 were never intubated, 2301 were intubated in the ED, and 1797 were intubated out-of-hospital. There must have been patient differences that prompted the paramedics, emergency physicians and admitting physicians to perform immediate, delayed or no intubation. Unfortunately, these differences are not captured in this kind of database; hence it remains unclear whether prehospital intubation or patient severity is the more important determinant of outcome. Other factors not captured or discussed included length of transportation times or need for extrication. Additional key information that has been recorded in other studies includes the number of intubation attempts, failed or misplaced endotracheal tubes, evidence of hypoxic periods during intubation, and the number of times tubes were dislodged en route. These additional data would further clarify the potential impact of procedural errors on patient outcomes.

Study results are probably applicable to our setting given that $88 \%$ suffered blunt trauma, similar to Canadian statistics. In addition, the data were derived from the entire state, not just urban or rural areas, including varying prehospital support systems and response times.

Importantly, as with any retrospective analysis, causality cannot be inferred. One can conclude that, after severe traumatic brain injury, out-of-hospital endotracheal intuba- 
tion is associated with higher mortality and worse neurologic and functional outcomes - not necessarily that one leads to the other. Now that several ${ }^{4-6}$ retrospective studies have identified prehospital intubation as a potential risk for increased mortality, investigators have identified the need for a well designed prospective trial to properly address the question of causality. ${ }^{10}$

Paramedicine, like medicine, often introduces new procedures and protocols based on expert consensus rather than clear evidence. EMS systems are extremely important but costly to run. It is critical that, in addition to education and implementation, valid prehospital research is carried out to ensure that widely accepted practices are leading to optimal patient outcomes. Determining the indications, safest techniques and maintenance of competency requirements for techniques like prehospital airway management and ventilation support are of great importance to the EMS and emergency medicine communities alike.

\section{Competing interests: None declared.}

\section{References}

1. Biros MH, Heegaard W. Prehospital and resuscitative care of the head-injured patient. Curr Opin Crit Care 2001;7:444-9.

2. Ochs M, Davis D, Hoyt D, et al. Paramedic-performed rapid sequence intubation of patients with severe head injuries. Ann Emerg Med 2002;40:159-67.

3. Winchell RJ, Hoyt DB. Endotracheal intubation in the field im- proves survival in patients with severe head injury: Trauma Research and Education Foundation of San Diego. Arch Surg 1997;132:592-7.

4. Murray JA, Demetriades D, Berne TV, et al. Prehospital intubation in patients with severe head injury. J Trauma 2000;49:1065-70.

5. Bochicchio GV, Ilahi O, Joshi M, et al. Endotracheal intubation in the field does not improve outcome in trauma patients who present without an acutely lethal traumatic brain injury. J Trauma 2003;54:307-11.

6. Davis DP, Hoyt DB, Ochs M, et al. The effect of paramedic rapid sequence intubation on outcome in patients with severe traumatic brain injury. J Trauma 2003;54:444-53.

7. Stiell IG, Wells GA, Field B, et al. Advanced cardiac life support in out-of-hospital cardiac arrest. N Engl J Med 2004;351: 647-56.

8. Gausche M, Lewis RJ, Stratton SJ, et al. Effect of out-of-hospital pediatric endotracheal intubation on survival and neurological outcome: a controlled clinical trial. JAMA 2000;283:783-90.

9. Newgard CD, Hedges JR, Arthur M, et al. Advanced statistics: the propensity score - a method for estimating treatment effect in observational research. Acad Emerg Med 2004;11:953-61.

10. DiRusso SM, Sullivan T, Risucci D, et al. Intubation of pediatric trauma patients in the field: predictor of negative outcome despite risk stratification. J Trauma 2005;59:84-90.

Correspondence to: Dr. Claude Topping, CCFP(EM) Program Director, Faculty of Medicine, Laval University, Quebec City QC G1K 7P4; fax 418 656-5252, claude.topping@mfa.ulaval.ca 